\title{
Strategie zum risikostratifizierten Einsatz von Antigen- Schnelltests: Eindämmung der SARS-CoV-2-Pandemie durch die Integration von Schnelltests in das Fall- und Kontaktpersonenmanagement
}

\author{
Strategy for a Risk-stratified Use of Rapid Antigen Testing: \\ Containing the SARS-CoV-2 Pandemic by Integrating Rapid Testing \\ into Case and Contact Tracing Management
}

\author{
Autoren \\ Laura Arnold ${ }^{*}$, Jan Stratil2* \\ Institute \\ 1 Akademie für Öffentliches Gesundheitswesen, \\ Düsseldorf, Deutschland \\ 2 Institut fur medizinische Informationsverarbeitung \\ Biometrie und Epidemiologie, Ludwig-Maximilians- \\ Universität München, München, Deutschland
}

\section{Schlüsselwörter}

COVID-19, Strategie, Schnelltests, Antigentest, Öffentlicher Gesundheitsdienst, Fall- und Kontaktpersonenmanagement

\section{Key words}

COVID-19, strategy, rapid testing, antigen testing, public health service, contact tracing

online publiziert $\quad 08.04 .2021$

\section{Bibliografie}

Gesundheitswesen 2021; 83: 349-353

DOI 10.1055/a-1408-3885

ISSN $0941-3790$

(c) 2021. Thieme. All rights reserved.

Georg Thieme Verlag KG, Rüdigerstraße 14,

70469 Stuttgart, Germany

Korrespondenzadresse

Laura Arnold

Akademie für Öffentliches Gesundheitswesen

Kanzlerstraße 4

40472 Düsseldorf

Deutschland

arnold@akademie-oegw.de

\section{ZUSAMMENFASSUNG}

Der Einsatz von Schnelltests bietet Chancen in der Bekämpfung der SARS-CoV-2 Pandemie; jedoch müssen die Auswirkungen von falsch-positiven und falsch-negativen Testergebnissen und die Reaktion der Bevölkerung antizipiert und berücksichtigt werden, um Schaden zu vermeiden. Auch geht ein ungezielter Einsatz von Schnelltests mit hohen direkten und indirekten Kosten einher und wird bei einem ineffizienten Ressourceneinsatz begrenzte Auswirkungen auf das Pandemiegeschehen haben können. Eine risikostratifizierte Teststrategie kann bei einer direkten Verknüpfung mit dem Fall- und Kontaktpersonenmanagement des Öffentlichen Gesundheitsdienstes (ÖGD) dazu beitragen, ressourceneffizient und nachhaltig die Infektionszahlen zu senken. Die Strategie sollte eine einheitliche Kommunikationsstrategie zum Umgang mit positiven und negativen Testergebnissen, eine gezielte Ausweitung der Zugänge zu niederschwelligen Testmöglichkeiten, die Sicherstellung eines zeitnahen und kostenlosen Zugangs zu den Ergebnissen von Bestätigungstests und die Einbindung in ein übergreifendes Dokumentationssystems zur Evaluation umfassen. Im Rahmen eines risikostratifizierten Einsatzes der Schnelltests sollten alle Personen mit akuten Symptomen einer Atemwegsinfektion sowie Personengruppen mit erhöhtem Risiko für das Bestehen einer SARS-CoV-2 Infektion gezielt getestet werden um „versteckte“ Infektionsnetzwerke zu identifizieren.

\section{ABSTRACT}

The use of rapid testing offers an opportunity to contain the SARS-CoV-2 pandemic; however, the impact of false-positive and false-negative test results and population response must be anticipated and taken into consideration to avoid or mitigate harm. Untargeted use of rapid testing is associated with high direct and indirect costs and will have limited impact on the pandemic if resources are used inefficiently. We suggest using a risk-stratified testing strategy, based on targeted testing directly integrated with the Public Health Service's case and contact tracing management. According to the proposed targeted testing strategy stratified by risk of infection, all persons with acute symptoms of a respiratory infection as well as other population groups with an elevated probability of being infected 
with SARS-CoV-2 infection should be specifically tested to identify "hidden" infection networks. The strategy should include a uniform communication strategy for dealing with positive and negative test results, a targeted expansion of access to low-threshold testing opportunities, ensuring timely and free access to the results of confirmatory tests, and integration into an overarching documentation system for evaluation. This integration of a risk-stratified targeted testing strategy into case and contact tracing management embedded in a comprehensive strategy can help to reduce infection rates in a resource-efficient and sustainable manner.

\section{Ausgangslage}

\section{Chancen und Risiken infolge der Test-Charakteristika}

Antigen-Schnelltests bieten zur Eindämmung der SARS-CoV-2-Pandemie große Chancen, da sie kostengünstig und schnell einen Großteil der Fälle mit einer hohen Viruslast entdecken können [1]. Vor allem wiederholtes Testen bietet die Chance, Ausbrüche zu kontrollieren [2-4].

Antigen-Schnelltests sind jedoch in ihrer Möglichkeit, infizierte Personen zu identifizieren limitiert[1]:

a) ein relevanter Anteil von infektiösen Personen wird nicht erfasst (von ca. einer Person aus fünf - also $20 \%$ bis zu einer Person aus 20 - also 5\%) [5-16]

b) die Sensitivität der Tests kann bei Kindern und Personen ohne Symptome (z. B. bei atypischem Verlauf oder während der präsymptomatischen Phase) deutlich niedriger ausfallen [6, 7, 14, 17-18]

c) das Testergebnis eines Antigen-Schnelltest stellt lediglich eine Momentaufnahme dar und ist in der Regel nur wenige Stunden, maximal einen Tag, gültig [3,19-21]

Zudem liefern Antigen-Schnelltests in deutlich höherem Maße als PCR-Tests falsch-positive Ergebnisse. Dies wird vor allem dann ein Problem, wenn viele Personen getestet werden, die eine geringe Wahrscheinlichkeit haben infiziert zu sein [5, 7, 8, 16, 18, 22, 23].

\section{INFOBOX: AUSMASS EINES FALSCH-POSITIVEN TESTERGEBNISSES}

Wenn in einer Stadt mit 100000 Einwohner/innen alle Personen bei einer Prävalenz von 35 pro 100000 mit einem Antigen-Schnelltests mit sehr guten Test-Charakteristika (Sensitivität 95\%, Spezifität 99,5\%) getestet werden würden, dann sind 533 positive Testergebnisse zu erwarten. Von diesen sind 33 Personen, die mit SARS-CoV-2 infiziert sind und 500 falsch positive Testergebnisse; was 93,8\% aller positiven Testergebnisse entspricht.

Des Weiteren muss berücksichtigt werden, dass die Güte der Antigen-Schnelltests, die sich in Deutschland aktuell auf dem Markt befinden, vielfach unklar ist. Die Angaben in der MedizinprodukteListe zum Antigen-Test auf SARS-CoV-2 des BfArM beruhen auf einem Abgleich der Herstellerangaben mit den durch das PEl in Abstimmung mit dem RKI festgelegten Mindestkriterien. Herstellerunabhängige Untersuchungen liefern z. T. deutlich abweichende (niedrigere) Ergebnisse, die durch Zufall allein nicht erklärt werden können [5-16, 18, 22, 23]. Hinzu kommt, dass die meisten Studien aufgrund eines geringen Stichprobenumfangs oft keine validen Aussagen über die Spezifität und damit die Rate falsch-positiver Testergebnisse treffen können.

Damit Antigen-Schnelltests einen entscheidenden Mehrwert zur Eindämmung der SARS-CoV-2-Pandemie liefern können, muss der Einsatz an die genannten Herausforderungen angepasst werden.

\section{Akzeptanz innerhalb der Bevölkerung}

Nutzen und Schaden eines großfächigen Einsatzes von AntigenSchnelltests hängen entscheidend vom Wissen, der Gesundheitskompetenz und dem Verhalten der Bevölkerung ab. So besteht das Risiko, dass die Anzahl an Neuinfektionen ansteigt, wenn infizierte Personen die Testsensitivität oder die Dauer der Aussagekraft eines negativen Testergebnisses überschätzen und sich infolge eines daraus resultierenden überhöhten Sicherheitsgefühls weniger an die $\mathrm{AHA}+\mathrm{L}$-Regeln halten oder vermehrt Risikoverhaltensweisen eingehen. Des Weiteren birgt eine hohe Anzahl falsch-positiver Testergebnisse das Risiko, dass die Aussagekraft positiver Testergebnisse generell als gering erachtet wird, was wiederum die Bereitschaft zum Nutzen von Schnelltests sowie die Bereitschaft zur vorgesehenen Isolation bis zur Verfügbarkeit eines PCR-Bestätigungstests erheblich verringern könnte.

Ein breiter Einsatz von Antigen-Schnelltests erfordert eine umfangreiche Kommunikationsstrategie zur Steigerung und Aufrechterhaltung der Akzeptanz innerhalb der Bevölkerung. Hierbei müssen auch Änderungen im (Risiko-)Verhalten der Bevölkerung antizipiert und berücksichtigt werden.

\section{Ressourceneinsatz und Adressat/innen}

Eine Teststrategie, die auf den bevölkerungsweiten Einsatz von Antigen-Schnelltests fokussiert (z. B. im Rahmen von Eigentestungen) oder Gruppen mit einem vergleichsweise geringen Infektionsrisiko regelmäßig testet (z. B. im Rahmen einer wöchentlichen Testung aller Schüler/innen und Lehrer/innen in Deutschland) geht mit einem massiven Ressourcenaufwand einher. Ressourcen, die primär auf nicht infizierte Personen und Personen mit einem geringen Infektionsrisiko zurückfallen und somit nur geringfügig zur Eindämmung der pandemischen Lage beitragen. 
INFOBOX: WAHRSCHEINLICHKEIT EINER SARSCOV-2 INFEKTION MIT UND OHNE RISIKO

Die Wahrscheinlichkeit nicht mit SARS-CoV-2 infiziert zu sein, liegt bei einer Person ohne bestehenden Risikokontakt und ohne Krankheitssymptome selbst in Regionen mit einer Prävalenz von 100 Infizierten pro 100000 bei>99,99\%. Wenn jedoch nur Personen betrachtet werden, die Symptome einer akuten Atemwegsinfektion aufweisen - und dies bei insgesamt 1500 Personen einer 100000 Einwohnerstarken Stadt der Fall wäre (Prävalenz =1,5\%), beträgt die Wahrscheinlichkeit einer symptomatischen Person mit SARS-CoV-2 infiziert zu sein ca. $6 \%$, was bei 1500 symptomatischen Personen 90 Infizierten entspricht.

Hinzu kommt, dass der ungezielte Einsatz von Testressourcen mit schwerwiegenden sozialen Konsequenzen einhergeht. Erkenntnisse aus der sozialepidemiologischen Forschung legen nahe, dass vor allem sozial besser gestellte Personen, Personen mit einem vergleichsweise geringen Infektionsrisiko und Personen mit einem insgesamt höheren Gesundheitsbewusstsein die Möglichkeit kostenloser Antigen-Schnelltests in Anspruch nehmen werden. Strukturell bedingt benachteiligte Personen, Personen mit einem erhöhten Infektionsrisiko sowie Personen mit einem insgesamt geringeren Gesundheitsbewusstsein werden das Angebot erwartungsgemäß deutlich seltener in Anspruch nehmen [24-26].

Eine umfassende Strategie zum bevölkerungsweiten Einsatz von Antigen-Schnelltests muss daher gleichermaßen die wirtschaftlichen Folgen des erwartbar hohen Ressourceneinsatzes sowie soziale Aspekte hinsichtlich schwer erreichbarer Bevölkerungsgruppen in den Blick nehmen.

\section{Lösungsansatz: Adaptiver Einsatz von Schnelltests}

Um den genannten Herausforderungen gerecht zu werden, muss der bevölkerungsweite Einsatz von Antigen-Schnelltests adaptiv erfolgen. Eine entsprechende Teststrategie sollte zwei Ansätze umfassen:

\section{Ziele einer risikostratifizierten Teststrategie}

a) Um die Infektionszahlen bundesweit langfristig senken zu können, müssen Personen mit einer erhöhten Wahrscheinlichkeit, mit SARS-CoV-2 infiziert zu sein, gezielt getestet und nach einem bestätigten positiven Testergebnis in die Kontaktpersonennachverfolgung eingebunden werden, um Infektionsketten möglichst frühzeitig unterbrechen zu können („Gezieltes Testen“)

b) Um Personen mit einem hohen Risiko für schwere Krankheitsverläufe vor den gravierenden Folgen einer SARS-Cov-2-Infektion zu schützen, müssen Testungen setting- und zielgruppenspezifisch erfolgen („Protektives Testen“)

Eine risikostratifizierte Teststrategie umfasst sowohl gezielte sowie protektive Testungen, je nach Bedarf. Die Umsetzung eines solchen adaptiven Ansatzes kann wie folgt gelingen:

\section{Einbringung in eine umfassende Gesamtstrategie}

Der Einsatz von Schnelltests muss in eine umfassende Gesamtstrategie eingebunden sein, bestehend aus:

(i) der Einbindung in die Arbeit des öffentlichen Gesundheitsdienstes (ÖGD) zum Fall- und Kontaktpersonenmanagement

Diese Einbindung ist der Grundpfeiler der Strategie, welche primär ihre Wirksamkeit durch die direkte Integration in die Kontaktpersonennachverfolgung entfaltet. Um weitere Infektionen durch positiv getestete Person und deren Kontaktnetzwerke zu verhindern, ist eine zeitnahe Reaktion notwendig.

(ii) einer risikostratifizierten Herangehensweise, die das mehrfache Testen von Personen mit einer erhöhten Wahrscheinlichkeit für das Bestehen einer SARS-CoV-2 Infektion ebenso ermöglicht wie das tägliche Testen zum Schutz von Personen mit einem hohen Risiko für schwere Krankheitsverläufe

Die Risikostratifizierung zielt darauf ab, begrenzte Ressourcen an den Punkten einzusetzen, wo sie den höchsten Nutzen bringen.

(iii) einer einheitlichen Kommunikationsstratgie

Diese sollte sowohl aus einer bevölkerungsweiten sowie zielgruppen- und kultursensiblen Aufklärungs- und Informationskampagne und einheitlichen Verfahrensanweisungen für alle beteiligte Akteur/innengruppen bestehen. Die Testzentren und Standorte für eine aktive Aufklärung der Aussagekraft und Begrenzungen der Testergebnisse genutzt werden.

(iv) der gezielten Ausweitung der Zugänge zu niederschwelligen Testmöglichkeiten für die gesamte Bevölkerung, insbesondere für vulnerable und schwer zugängliche Personengruppen

Eine mehrmalige Testung bei leichten Symptomen setzt einen alltagsnahen und niederschwelligen Zugang zu Testmöglichkeiten voraus. Neben der Einrichtung zentral gelegener Testeinrichtungen, sollte hierzu auch die Nutzung bestehender Infrastruktur wie Apotheken oder Arztpraxen und der Aufbau aufsuchender Teams, um beispielsweise Testungen in Schulen oder Betrieben realisieren zu können, geprüft werden. Dabei muss insbesondere die Erreichbarkeit von Personen mit eingeschränkter Mobilität berücksichtigt werden.

(v) der Sicherstellung eines zeitnahen, kostenlosen und niedrigschwelligen Zugangs zu den Ergebnissen von Bestätigungstests

Durch die hohe Rate falsch-positiver Testergebnisse muss jedes positive Schnelltestergebnis mittels eines PCR-Tests geprüft werden. Grundlage für eine Schnellteststrategie sollte daher das Bestehen einer Infrastruktur sein, die den raschen Zugang zu den Ergebnissen eines Bestätigungstest garantiert. 
(vi) der Etablierung eines einheitlichen und übergreifenden Informationssystems, um die flächendeckende Surveillance sämtlicher positiver Testergebnisse sicherzustellen

Hier bietet sich eine bundesweit einheitliche Onlineplattform, auf welche nicht nur alle Testzentren und Teststandorte medienbruchfrei zugreifen können, sondern darüber hinaus auch die Ergebnisse der Schnelltests sowie die bei positivem Testergebnis notwendigen Bestätigungstests unkompliziert registriert werden können. Auf diese Weise können Reichweite und Testgüte live evaluiert werden.

\section{Gezieltes Screening}

Durch gezieltes Screening können „versteckte“ Infektionsherde (d. h. Netzwerke von Infektionen, die keinem Ausbruch oder bekannten Infektionsnetzwerk zugeordnet werden können) identifiziert und diese rasch und gezielt der kommunalen Kontaktpersonennachverfolgung zugeführt warden [27, 28]. Die Fokussierung auf Personengruppen mit einer erhöhten Wahrscheinlichkeit für das Bestehen einer SARS-CoV-2 Infektion ermöglicht ein gezieltes Ressourcenmanagement. Im Rahmen des gezielten Screenings sollten alle Personen mit Symptomen einer akuten Atemwegsinfektion sowie Kontaktpersonen der Kategorie I und II gezielt getestet werden und bei einem PCR-positiv bestätigten Testergebnis direkt in die Kontaktpersonennachverfolgung eingebunden werden. Hierzu sind klare und einfache Vorgaben an die Bevölkerung genauso wie eine entsprechende Testinfrastruktur notwendig.

\section{INFOBOX: RESSOURCENEINSATZ EINES GEZIELTEN SCREENINGS}

Weisen in der bereits zuvor genannten Stadt mit 100000 Einwohner/innen bei einer Prävalenz von 35 pro 100000 Personen (80\% symptomatischen Verläufen) rund $1,5 \%$ der Bevölkerung Symptome einer Atemwegsinfektion auf, so trifft dies auf 1500 Personen zu. Werden nun alle 1500 symptomatischen Personen im Verlauf des Bestehens der Symptome 3-mal getestet (Testsensitivität 95\%), würden mit 4500 Tests 27 der 35 infizierten Personen erfasst. Wenn nur eine Person aus 7 nicht-symptomatische Personen in Ihrem Kontaktnetzwerk hat, könnte der Ausbruch mit maximaler Ressourceneffizienz durch die Kombination aus gezielten Schnelltest-Screenings und Kontaktpersonennachverfolgung kontrolliert werden.

\section{Intelligentes Ausbruchsmanagement:}

In Regionen und Settings mit hohen Infektionsraten und/oder hohen Risiken für Folgeinfektionen oder größeren Ausbrüchen, bietet sich eine gezielte Testung aller Personen in mehreren Sequenzen an, bis kein weiterer Infektionsfall mehr identifiziert wird $[2,4]$. Dies kann sowohl Kommunen mit einer sehr hohen Infektionslast (z. B. 7-Tages Inzidenz > 250/100 000 EW) als auch Schulen oder Betriebe mit einem entsprechendem Ausbruchsgeschehen umfassen.

\section{Protektive Testungen zum Schutz von Risikogruppen:}

In Settings mit besonders hohem Risiko für Ausbrüche und gravierenden Konsequenzen von Infektionen, wie es z. B. in Altenund Pflegeheimen ohne ausreichenden Impfschutz häufig der Fall ist, sollte der Zugang durch tägliches Testen aller aufsuchenden Personen, wie z. B. Pflegepersonal oder Besucher/innen, kontrolliert werden [29-32].

\section{Stärken einer risikostratifizierten Teststrategie}

Bei einem risikostratifizierten Einsatz von Antigen-Schnelltests werden begrenzte Ressourcen gezielt und effektiv zur Senkung der Infektionszahlen in der Bevölkerung sowie gleichermaßen zum Schutz von Risikopersonen eingesetzt.

Die Schwächen bei Test-Sensitivität werden durch ein wiederholtes Testen erheblich reduziert [2-4]. Zudem fokussiert die Gesamtstrategie nicht auf die Identifikation einer jeder einzelnen Infektion, sondern primär auf die zu erwartenden positiven Effekte auf Bevölkerungsebene. Bei der gezielten Verbindung des risikostratifizierten Einsatzes von Schnelltests mit einer direkten Nachverfolgung der jeweiligen Kontaktnetzwerke erhöht sich die Chance, dass zeitnah weitere Fälle aufgedeckt werden können.

Durch die deutlich höhere Vortestwahrscheinlichkeit eines risikostratifizierten Testens fällt der Anteil falsch-positiver Testergebnisse deutlich geringer aus und insgesamt weniger ins Gewicht [1]. Die weitreichenden Konsequenzen falsch-positiver Testergebnisse können ferner durch die zeitnahe Realisierung von Bestätigungstests reduziert werden, welche innerhalb von $24 \mathrm{~h}$ erfolgen sollten.

Ein intelligenter Einsatz von Antigen-Schnelltests bietet zahlreiche Synergien zur Arbeit des öffentlichen Gesundheitsdiensts (ÖGD) und lässt sich direkt in das Fall- und Kontaktpersonenmanagement integrieren. Als größter Public Health-Akteur vor Ort kann der ÖGD bei der Identifikation gefährdeter Bevölkerungsgruppen ebenso behilflich sein, wie bei der Identifikation relevanter Settings.

Durch ein einheitliches, übergreifendes Informationssystem können bestehende Wissenslücken bezüglich der Testcharakteristika geschlossen und ungeeignete Testsysteme identifiziert und in der Folge vom Markt genommen werden.

Interessenkonflikt

Alle Autorlnnen erklären, dass kein wirtschaftlicher oder persönlicher Interessenkonflikt vorliegt. LA ist als Referentin für Epidemiologie und Gesundheitsberichterstattung an der Akademie für Öffentliches Gesundheitswesen tätig.

\section{Literatur}

[1] Seifried J, Böttcher S, Oh D et al. Was ist bei Antigentests zur Eigenanwendung (Selbsttests) zum Nachweis von SARS-CoV-2 zu beachten? Epidemiologisches Bulletin 2021; 8: 3-9. doi: $10.25646 / 8040$

[2] Larremore DB, Wilder B, Lester $E$ et al. Test sensitivity is secondary to frequency and turnaround time for COVID-19 screening. 2021; 7: eabd5393 doi: 10.1126/sciadv.abd5393 \%] Science Advances 
[3] Mina M], Parker R, Larremore DB. Rethinking Covid-19 Test Sensitivity - A Strategy for Containment. 2020; 383: e120. doi: 10.1056/ NEJMp2025631

[4] Paltiel AD, Zheng A, Walensky RP. Assessment of SARS-CoV-2 Screening Strategies to Permit the Safe Reopening of College Campuses in the United States. JAMA network open 2020; 3 : e2016818-e2016818. doi: 10.1001/jamanetworkopen.2020.16818

[5] Abdulrahman A, Mustafa F, AlAwadhi Al et al. Comparison of SARS-COV-2 nasal antigen test to nasopharyngeal RT-PCR in mildly symptomatic patients. 2020: 2020.11.10.20228973. doi: 10.1101/2020.11.10.20228973\%] medRxiv

[6] Paul G, Plecko T, Sethi S et al. Klinische Performance eines neuen SARS-CoV-2-AntigenTests in der Notaufnahme eines Maximalversorgers. Epidemiologisches Bulletin 2021; 3: 10-15

[7] Berger A, Ngo Nsoga MT, Perez-Rodriguez F] et al. Diagnostic accuracy of two commercial SARS-CoV-2 Antigen-detecting rapid tests at the point of care in community-based testing centers. 2020:2020.11.20.20235341. doi: 10.1101/2020.11.20.20235341 \%] medRxiv

[8] Alemany A, Baró B, Ouchi D et al. Analytical and clinical performance of the panbio COVID-19 antigen-detecting rapid diagnostic test. Journal of Infection 2020. doi: 10.1016/j.jinf.2020.12.033

[9] Schwob JM, Miauton A, Petrovic D et al. Antigen rapid tests, nasopharyngeal PCR and saliva PCR to detect SARS-CoV-2: a prospective comparative clinical trial. 2020:2020.11.23.20237057. doi: 10.1101/2020.11.23.20237057\%] medRxiv

[10] Osterman A, Baldauf H-M, Eletreby $M$ et al. Evaluation of two rapid antigen tests to detect SARS-CoV- 2 in a hospital setting. Medical Microbiology and Immunology 2021. doi: 10.1007/s00430-02000698-8

[11] Lindner AK, Nikolai O, Rohardt C et al. Head-to-head comparison of SARS-CoV-2 antigen-detecting rapid test with professional-collected nasal versus nasopharyngeal swab. 2021:2020.12.03.20243725. doi: 10.1101/2020.12.03.20243725 \%] medRxiv

[12] Lindner AK, Nikolai O, Rohardt C et al. SARS-CoV-2 patient self-testing with an antigen-detecting rapid test: a head-to-head comparison with professional testing. 2021:2021.01.06.20249009. doi: 10.1101/2021.01.06.20249009\%] medRxiv

[13] Cerutti F, Burdino E, Milia MG et al. Urgent need of rapid tests for SARS CoV-2 antigen detection: Evaluation of the SD-Biosensor antigen test for SARS-CoV-2. Journal of clinical virology: the official publication of the Pan American Society for Clinical Virology 2020; 132: 104654. doi: 10.1016/j.jcv.2020.104654 [published Online First: 2020/10/15]

[14] Linares M, Pérez-Tanoira R, Carrero A et al. Panbio antigen rapid test is reliable to diagnose SARS-CoV- 2 infection in the first 7 days after the onset of symptoms. Journal of Clinical Virology 2020; 133: 104659. doi: https://doi.org/10.1016/j.jcv.2020.104659

[15] Hoehl S, Schenk B, Rudych O et al. At-home self-testing of teachers with a SARS-CoV-2 rapid antigen test to reduce potential transmissions in schools. Results of the SAFE School Hesse Study. 2020; 2020.12.04.20243410. doi: $10.1101 / 2020.12 .04 .20243410 \%]$ medRxiv

[16] Dinnes ], Deeks J], Adriano A et al. Rapid, point-of-care antigen and molecular-based tests for diagnosis of SARS-CoV-2 infection. The Cochrane database of systematic reviews 2020; 8: Cd013705. doi: 10.1002/14651858.Cd013705 [published Online First: 2020/08/28]

[17] Pollock NR, Savage T], Wardell $\mathrm{H}$ et al. Correlation of SARS-CoV-2 nucleocapsid antigen and RNA concentrations in nasopharyngeal samples from children and adults using an ultrasensitive and quantitative antigen assay. 2021; JCM.03077-20. doi: 10.1128/ JCM.03077-20 \%] Journal of Clinical Microbiology
[18] Bulilete O, Lorente P, Leiva A et al. Evaluation of the Panbio ${ }^{\mathrm{TM}}$ rapid antigen test for SARS-CoV-2 in primary health care centers and test sites. 2020:2020.11.13.20231316. doi: 10.1101/2020.11.13. $20231316 \%$ J medRxiv

[19] He X, Lau EHY, Wu P et al. Temporal dynamics in viral shedding and transmissibility of COVID-19. Nature Medicine 2020; 26: 672-675. doi: 10.1038/s41591-020-0869-5

[20] Kucirka LM, Lauer SA, Laeyendecker O et al. Variation in False-Negative Rate of Reverse Transcriptase Polymerase Chain Reaction-Based SARS-CoV-2 Tests by Time Since Exposure. Annals of internal medicine 2020; 173: 262-267. doi: 10.7326/m20-1495 [published Online First: 2020/05/19]

[21] Mallett S, Allen A], Graziadio S et al. At what times during infection is SARS-CoV-2 detectable and no longer detectable using RT-PCR-based tests? A systematic review of individual participant data. BMC medicine 2020; 18. doi: 10.1186/s12916-020-01810-8

[22] Corman VM, Haage VC, Bleicker T et al. Comparison of seven commercial SARS-CoV-2 rapid Point-of-Care Antigen tests. 2020:2020.11.12.20230292. doi: 10.1101/2020.11.12.20230292 \%] medRxiv

[23] Iglói Z, Velzing J, van Beek J et al. Clinical evaluation of the Roche/SD Biosensor rapid antigen test with symptomatic, non-hospitalized patients in a municipal health service drive-through testing site. 2020:2020.11.18.20234104. doi: 10.1101/2020.11.18.20234104\%] medRxiv

[24] Janßen C, Frie K, Dinger H et al. Der Einfluss von sozialer Ungleichheit auf die medizinische und gesundheitsbezogene Versorgung in Deutschland. I. In: Richter M, Hurrelmann K eds. Gesundheitliche Ungleichheit: Grundlagen, Probleme, Perspektiven. $2^{\text {nd }}$ Edition Wiesbaden: VS Verlag für Sozialwissenschaften; 2009: 149-165

[25] Jordan S, Krug S, Manz K et al. Health behaviour and COVID-19: Initial findings on the pandemic. 2020; (S8/20) 1-14. doi: http://dx.doi. org/10.25646/7055

[26] Wachtler B, Michalski N, Nowossadeck E et al. Socioeconomic inequalities and COVID-19 - A review of the current international literature. 2020; (S7) 3-17. doi: http://dx.doi.org/10.25646/7059

[27] Contreras S, Dehning J, Loidolt $\mathrm{M}$ et al. The challenges of containing SARS-CoV-2 via test-trace-and-isolate. Nature Communications 2021; 12: 378. doi: 10.1038/s41467-020-20699-8

[28] Kucharski A], Klepac P, Conlan AJK et al. Effectiveness of isolation, testing, contact tracing, and physical distancing on reducing transmission of SARS-CoV- 2 in different settings: a mathematical modelling study. The Lancet Infectious Diseases 2020; 20: 1151-1160. doi: 10.1016/S1473-3099(20)30457-6

[29] Smith DRM, Duval A, Pouwels KB et al. Optimizing COVID-19 surveillance in long-term care facilities: a modelling study. 2020; 18: 386

[30] Tsoungui Obama HC, Nessma Adil MY, Looli Alawam N et al. Preventing COVID-19 spread in closed facilities by regular testing of employees - an efficient intervention in long-term care facilities and prisons. 2020:2020.10.12.20211573. doi: 10.1101/2020.10. $12.20211573 \%$ ] medRxiv

[31] Holmdahl I, Kahn R, Hay J et al. Frequent testing and immunity-based staffing will help mitigate outbreaks in nursing home settings. 2020;

[32] See I, Paul P, Slayton RB et al. Modeling effectiveness of testing strategies to prevent COVID-19 in nursing homes - United States, 2020. Clinical Infectious Diseases 2021. doi: 10.1093/cid/ciab110 